Check for updates

Cite this: RSC Adv., 2017, 7, 43755

Received 27th July 2017

Accepted 29th August 2017

DOI: $10.1039 / \mathrm{c} 7 \mathrm{ra0} 272 \mathrm{j}$

rsc.li/rsc-advances

\title{
A high modulus hydrogel obtained from hydrogen bond reconstruction and its application in vibration damper†
}

\author{
Longxiang Zhu, Jianhui Qiu (D)* and Eiichi Sakai
}

\begin{abstract}
Hydrogels are wet and soft materials with rubber-like properties. The excellent biocompatibility and stimuliresponsiveness have made hydrogels excellent candidates in the field of materials science. However, most of the hydrogels are extremely soft (modulus of approximately $0.1 \mathrm{MPa}$ ) as compared to rubber materials; this greatly limits their application in the field of material engineering. In this study, an $\mathrm{Al}^{3+}$-reinforced carboxymethyl cellulose/polyacrylic acid hydrogel was first synthesized by a facile, visible-light-triggered, one-pot polymerization method. Subsequently, the as-prepared hydrogel was reinforced by evaporation-swelling (E-S) treatment to obtain a hydrogel (HM-Gel) with a 10 -fold higher elastic modulus. This hydrogel exhibits a tensile strength of 1.26-1.74 MPa and an elastic modulus of 0.591.94 MPa. Moreover, the HM-Gel, with an excellent vibration absorption ability, can find applications in the field of industrial engineering and bioengineering.
\end{abstract}

\section{Introduction}

Hydrogels are engineering materials with a high water content and exhibit a three-dimensional network structure. ${ }^{\mathbf{1 , 2}}$ Due to their inherently excellent biocompatibility and stimuli-responsiveness, hydrogels have been applied in the field of tissue engineering. ${ }^{3-5}$ In recent years, hydrogels incorporating effective energy dissipation mechanisms or presenting distinctive structures exhibit remarkable overall mechanical properties (tensile strength of 0.2-10 MPa; fracture elongation of $100-6000 \%$, and toughness of 100-15 $\left.000 \mathrm{~J} \mathrm{~m}^{-2}\right) .{ }^{6}$ These hydrogels, which exhibit similar properties to conventional rubbers, are excellent candidates for vibration control materials in tissue engineering. ${ }^{7}$ The vibration absorption material must have sufficient rigidity (to reduce the amplitude during vibration); therefore, this material must have a high modulus (e.g., cartilage, a natural vibration absorption material, has a modulus greater than $1 \mathrm{MPa}){ }^{8}$ However, the modulus of most hydrogels is of the order of $0.1 \mathrm{MPa},{ }^{9,10}$ which greatly limits their use as a vibration absorption material.

In recent years, researchers have developed a number of high-modulus hydrogels through novel design concepts and special structures. ${ }^{11}$ Gong et al. have designed an oppositely charged polyelectrolyte PMPTC/PNaSS hydrogel. ${ }^{12}$ In the

Department of Machine Intelligence and Systems Engineering, Faculty of Systems Engineering, Akita Prefectural University, Akita, 015-0055, Japan. E-mail: qiu@ akita-pu.ac.jp

$\dagger$ Electronic supplementary information (ESI) available. See DOI: $10.1039 / \mathrm{c} 7 \mathrm{ra0} 08272 \mathrm{j}$ preparation process, a uniformly dispersed polyelectrolyte hydrogel was obtained by a two-step polymerization reaction. The resulting hydrogel was dialyzed to ensure that the ionic bonds in the hydrogel were sufficiently strong. The highest modulus of the PMPTC/PNaSS hydrogel can reach 7.9 MPa. In addition, Lin et al. improved a dual-crosslinked hydrogel consisting of covalent and ionic bonds. ${ }^{13}$ In this hydrogel, the initial PAM-PAA network was synthesized using a covalent cross-linker, and then, the coordination bonds were formed after immersing the single network hydrogel in a highly concentrated $\mathrm{Fe}^{3+}$ solution. The modulus of the PAM/PAA hydrogel can reach 3.5 MPa. Moreover, optimization of the network structure in the as-prepared hydrogel is an effective way to increase the modulus. For instance, increasing the crystallinity of the polymer chains within the hydrogel by the addition of a salt or base solution at high concentration can enhance the modulus of the hydrogel. Thus, the modulus of the PAM-CS hydrogel can reach 0.3-0.4 MPa through crystallization enhancement; however, the use of a strong base may cause potential problems in applications. ${ }^{14}$ Therefore, it is a big challenge to produce a hydrogel with high modulus through a convenient, efficient, and green synthesis method. Most hydrogels contain a variety of complex hydrogen bond networks across polymer chains; these hydrogen bond interactions between polymer chains can be leveraged to achieve a reconstruction of the hydrogel network to improve the modulus of the hydrogel.

In our previous study, we prepared a carboxymethyl cellulose/polyacrylic acid hydrogel with excellent mechanical properties (tensile strength of $0.85 \mathrm{MPa}$, fracture elongation of $700 \%$, and modulus of $0.18 \mathrm{MPa}$ ) by a facile, visible-light- 
triggered, one-pot polymerization method. ${ }^{15,16}$ Herein, a aluminum ion cross-linked hydrogel $(\mathrm{Gel})$ was synthesized by the same method, and then, the as-prepared Gel was reinforced via the evaporation-swelling method (E-S method) to obtain the high-modulus hydrogels (HM-Gel), with a tensile strength of 1.26-1.74 MPa and an elastic modulus of 0.59-1.94 MPa. In addition, both Gel and HM-Gel exhibited improved damping performance due to the presence of aluminum ion as a reinforcement cross-linking site; thus, the applicability of the hydrogel as a vibration control material is greatly improved. This E-S method provides a way for increasing the modulus of many reported hydrogels and can broaden their application as a vibration control material.

\section{Materials and methods}

\section{Materials}

Carboxymethyl cellulose sodium salt (CMC, EP), acrylic acid (AA, EP), ceric ammonium nitrate $\left(\left(\mathrm{NH}_{4}\right)_{2} \mathrm{Ce}\left(\mathrm{NO}_{3}\right)_{4}, \mathrm{CAN}, \mathrm{GR}\right)$, and aluminium(III) chloride hexahydrate $\left(\mathrm{AlCl}_{3} \cdot 6 \mathrm{H}_{2} \mathrm{O}, \mathrm{GR}\right)$ were purchased from Nacalai Tesque, Inc. All chemical reagents were used as received. Distilled water was used in all experiments.

\section{Sample preparation}

The HM-Gels were prepared via two steps consisting of visiblelight-triggered polymerization and evaporation-swelling. Briefly, $40 \mathrm{mg}$ of CAN, $3.60 \mathrm{~g}$ of AA, and $10.0 \mathrm{~mL}$ of distilled water were added to a vial stepwise and mixed under magnetic stirring in an ice bath. Subsequently, $0.40 \mathrm{~g}$ of CMC was slowly added to the mixture under vigorous magnetic stirring. The precursor hydrogel was stably dispersed for $40 \mathrm{~min}$ under vigorous stirring. A range of concentrations of $\mathrm{AlCl}_{3} \cdot 6 \mathrm{H}_{2} \mathrm{O}$ $\left(0-1.5 \mathrm{~mol} \%, \mathrm{Al}^{3+}\right.$ to $\left.\mathrm{AA}\right)$ was added to a series of vials, and the mixtures were stirred for $20 \mathrm{~min}$. The viscous precursor hydrogels were transferred to transparent glass molds and covered with glass plates. Finally, the glass molds were immersed in water at $20^{\circ} \mathrm{C}$ and placed $20 \mathrm{~cm}$ below a visible light source (LS-M210, Sumita) for $5 \mathrm{~min}$. The prepared carboxymethyl cellulose/polyacrylic acid hydrogels were named Gel- $X$, where $X$ denoted the $\mathrm{Al}^{3+}$ concentration (mol\%) relative to that of monomer (AA). The obtained Gels were placed in a dry oven and evaporated at various temperatures for $24 \mathrm{~h}$ (for $25^{\circ} \mathrm{C}$, the hydrogel was evaporated at room temperature for 3 days). The dried Gels were immersed in distilled water for $50 \mathrm{~h}$ to obtain a swelling equilibrium. The swollen hydrogels at equilibrium with a high modulus were named HM-Gel. Unless otherwise specified, all hydrogels were evaporated in air at $60{ }^{\circ} \mathrm{C}$.

\section{Characterization}

The phase transition time of the hydrogels was determined by dynamic mechanical analysis (DMA, RSA-G2, TA Instrument, New Castle, DE, USA). The improved experimental set-up is shown below:

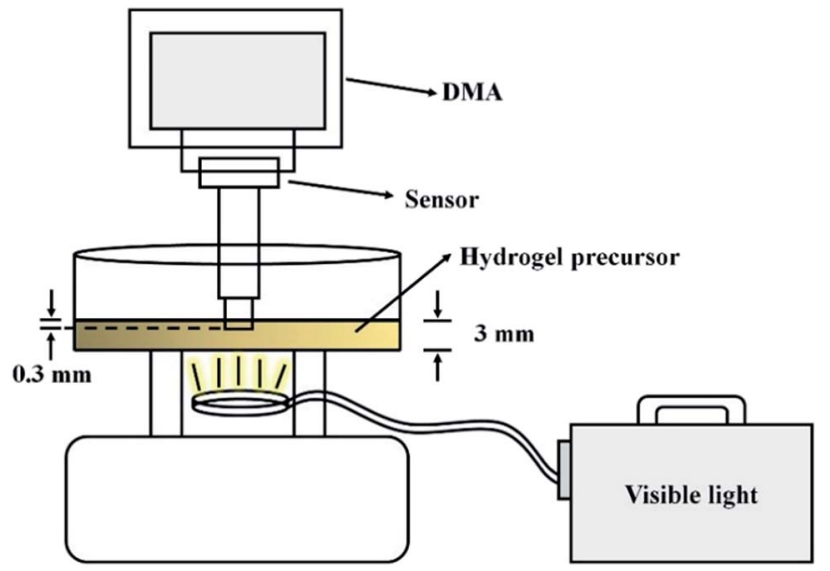

We used a stress relaxation modulus program for the hydrogel gelation time tests using a disc model at $25{ }^{\circ} \mathrm{C}, 1 \mathrm{~Hz}$, and $1 \%$ strain. The program and light were turned on at the same time.

The uniaxial elongation properties were measured using the JIS K6251 (a Japanese industrial rubber) as a standard via a universal testing machine (Instron 3300 series, $50 \mathrm{~N}$ load cell, Instron Co., Ltd., Canton, America); the procedure for the measurements was as follows: hydrogels were cut in a dumbbell shape with a $75 \mathrm{~mm}$ length $(l), 25 \mathrm{~mm}$ gauge length $\left(l_{\mathrm{o}}\right), 4 \mathrm{~mm}$ width $(w)$, and $2 \mathrm{~mm}$ height $(h)$, and tests were conducted at a cross-head speed of $500 \mathrm{~mm} \mathrm{~min}^{-1}$. The compression properties were measured using the same universal testing machine at a cross-head speed of $1 \mathrm{~mm} \mathrm{~min}^{-1}$. Hydrogels were cut in a disc shape with $9 \mathrm{~mm}$ diameter and $2 \mathrm{~mm}$ height.

The storage modulus and loss modulus of the hydrogel specimens were measured as a function of frequency using dynamic mechanical analysis (DMA, RSA-G2, TA Instrument). Hydrogels were cut into a disc shape with $9 \mathrm{~mm}$ diameter and $2 \mathrm{~mm}$ height. Before measurements, an axial force of $0.981 \mathrm{~N}$ was applied on the specimens. The frequency sweeping experiments were performed under a constant strain amplitude (1\%) in the frequency range from 100 to $0.1 \mathrm{~Hz}$.

For the cyclic tensile tests, loading-unloading measurements were performed using the same universal testing machine at a constant velocity of $100 \mathrm{~mm} \mathrm{~min}^{-1}$. The Gels were cut into pieces with the following size: $60 \mathrm{~mm}$ length $(l), 10 \mathrm{~mm}$ width $(w), 2 \mathrm{~mm}$ height $(h)$, and $30 \mathrm{~mm}$ gauge length $\left(l_{\mathrm{o}}\right)$. The samples were covered with oil to prevent water evaporation. Tensile stress $(\sigma)$ was calculated as $\sigma=F / w h$, where $F$ is the load.

Swelling experiments were performed by immersing the Gels and dried-Gels in conical flasks filled with distilled water. The flasks were placed in a temperature-controlled bath at $25{ }^{\circ} \mathrm{C}$ for 50 hours. The swelling ratio $\left(Q_{\mathrm{m}}\right)$ was calculated by the following equation:

$$
Q_{\mathrm{m}}=W_{\mathrm{s}} / W_{\mathrm{a}}
$$

where $W_{\mathrm{s}}$ is the weight of the swollen hydrogel and $W_{\mathrm{a}}$ is the weight of the as-prepared Gels. For the swelling experiment, all samples were measured 3 times.

The changes of the CMC crystalline network structure in Gel and HM-Gel were investigated using an optical microscope 
(Eclipse model ME 600D, Nikon, Japan). The thin hydrogel films were obtained by shock-freezing using a microtome (RM2145, Leica Microsystems, Japan). The length, width, and thickness of the hydrogel films were $15 \mathrm{~mm}, 2 \mathrm{~mm}$, and $200 \mu \mathrm{m}$, respectively.

Differential thermal analysis (DTA) of the samples was performed using a TG instrument (DTG-60, Shimadzu Co., Ltd., Kyoto, Japan) at a scan rate of $10{ }^{\circ} \mathrm{C} \mathrm{min}^{-1}$ from room temperature to $400{ }^{\circ} \mathrm{C}$ under a $\mathrm{N}_{2}$ atmosphere (flow rate of

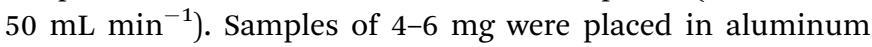
crucibles using an empty aluminum crucible as a reference.

To fully understand the changes in the internal network, we estimated the effective network chain density $(N)$ based on the cyclic stress-strain curves of HM-Gel (dried in air at $60{ }^{\circ} \mathrm{C}$ ), according to the following equation reported in the literature:

$$
\tau=N R T\left[\alpha-(1 / \alpha)^{2}\right]
$$

where $\tau$ is the force per unit of unstrained cross-sectional area, $R$ and $T$ are the gas constant and absolute temperature, respectively, and a $\tau$ value at elongation of $\alpha=2$ (strain 100\%) is used in the calculation.

For the experiments evaluating the damping performance, a commercially available test tube mixer was used as the vibration source; the vibration machine was equipped with a hydrogel vibration damper, a support plate, and a force sensor. The sampling frequency was set at 20 points per second.

All the hydrogel performance tests were performed at room temperature.

\section{Results and discussion}

\section{Visible-light intensity for hydrogel formation}

Light as an efficient and easily acquired trigger has been extensively used for radical-initiated polymerization. ${ }^{\mathbf{1 7}}$ Visible light, which is safe, low cost, and easy to use, is favored in synthetic polymerization applications. ${ }^{18}$ In our previous study, visible light was found to be an efficient trigger for the synthesis of cellulose-containing hydrogels. ${ }^{15}$ In this polymerization, ceric ions attack the glucopyranose units to form a CMC-cerium complex. Then, $\mathrm{Ce}^{4+}$ ions are reduced to $\mathrm{Ce}^{3+}$ within the complex, and a free radical and aldehyde are obtained by the rupture of the carbon 2 and 3 bonds of the glucopyranose unit. ${ }^{19}$ Finally, the free radical initiates the monomer polymerization and a network is formed through end-group termination of the active polymer chains (Scheme 1). This polymerization can even be performed under sunlight, and the gelation process is completed within only 1 minute (Fig. 1, inset). To study the effect of light intensity on hydrogel performance, a metal halide lamp (LS-M210, Sumita) was selected as a stable visible light source. The luminous emittance is proportional to the output energy. Fig. S1† shows that the luminous emittance was in the range of 58-215 kLux depending on the output energy (from $25 \%$ to $100 \%$ ). Since the generated radicals are highly dependent on the induction of visible light, the gelation time is closely related to the visible light intensity. The pseudo relaxation modulus showed that the hydrogel precursors from the viscous state to the just-formed solid state require $68 \mathrm{~s}, 30 \mathrm{~s}, 20 \mathrm{~s}$, and $22 \mathrm{~s}$ at the visible light output energy of $25 \%, 50 \%, 75 \%$, and $100 \%$, respectively (Fig. 1). This suggests that high light intensity helps to shorten the gelation time, but higher light intensity has a limited effect on the gelation time. Moreover, the visible light intensity greatly affects the mechanical properties of the hydrogel. At a lower output energy (25\%), the tensile strength, fracture elongation, and elastic modulus of Gel-1.0 were $0.59 \mathrm{MPa}, 640 \%$, and $0.096 \mathrm{MPa}$, respectively. When a higher output energy of $50 \%, 75 \%$, and $100 \%$ was applied, the tensile strength and elastic modulus of Gel-1.0 clearly improved (Fig. S2 $\dagger$ ). However, the fracture elongation slightly decreased with the increasing output energy.

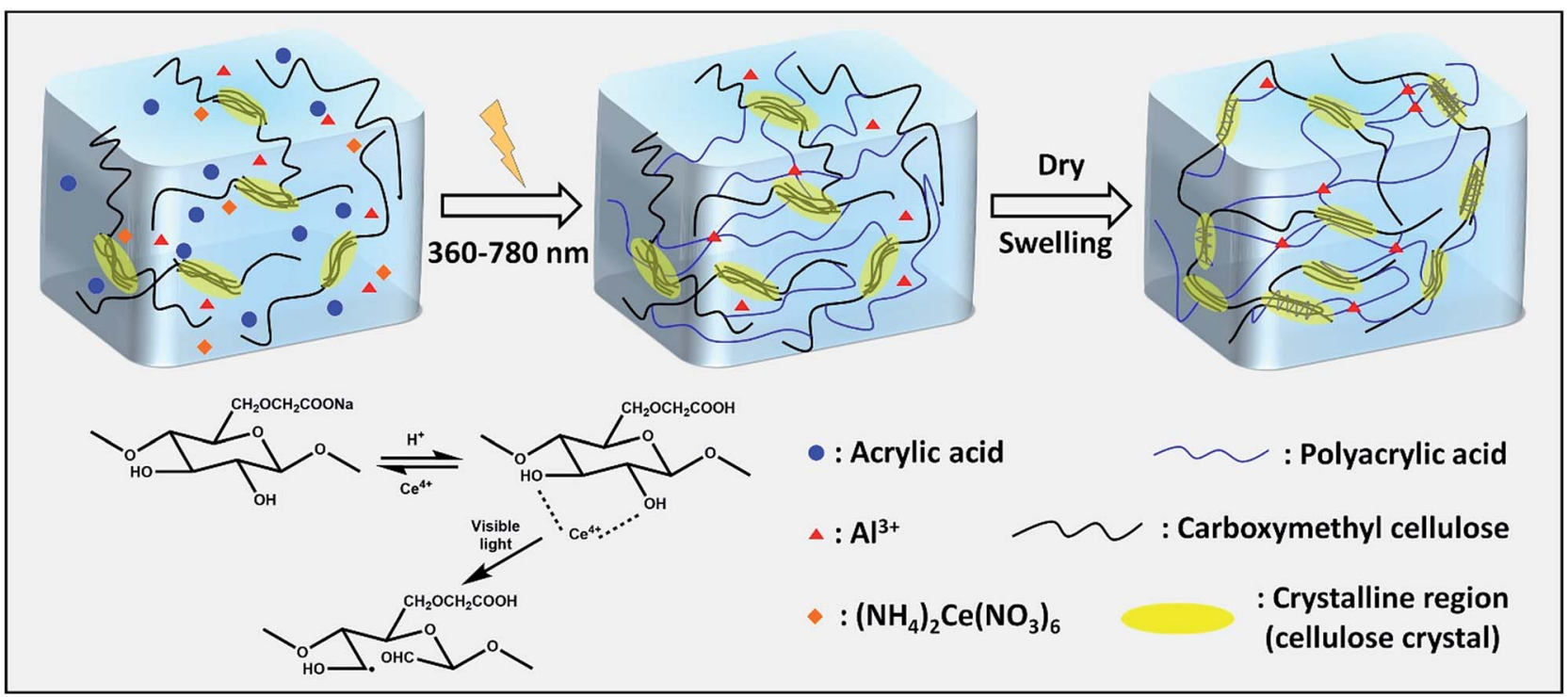

Scheme 1 Proposed mechanism for the formation of the hydrogel under visible light and the reinforced process by the evaporation-swelling method. 


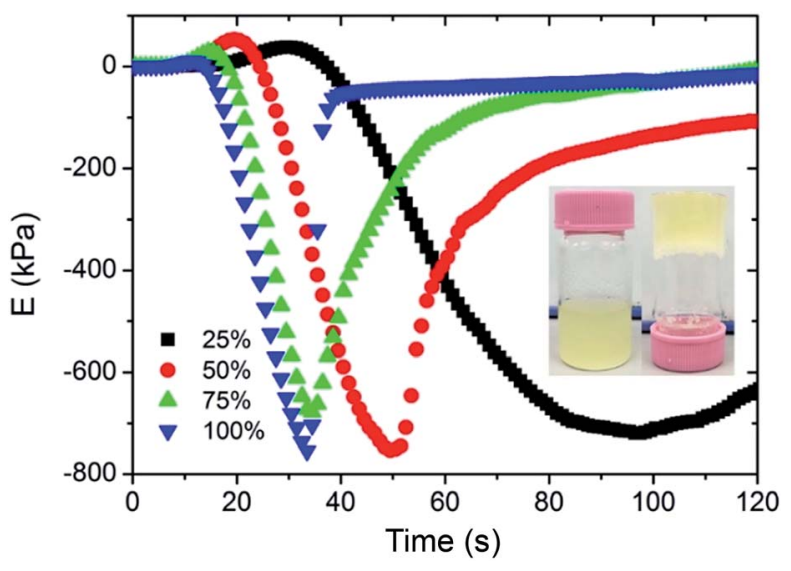

Fig. 1 Effect of various light intensities on the time of hydrogel state transformation: pseudo relaxation modulus-time curves of Gel-1.0. The inset is Gel-1.0 before and after polymerization under sun light.

\section{Improvement of mechanical properties}

To improve the mechanical performance of hydrogels, researchers have intensively investigated different reinforced networks including double networks, ${ }^{3}$ topological networks, ${ }^{20}$ polyampholyte based networks, ${ }^{21}$ hydrogen bond cross-linked networks, ${ }^{22}$ or ionic cross-linked networks. ${ }^{23}$ Among them, the ionic cross-linking is one of the most deeply investigated. Metal cations play an important role in hydrogels containing anionic polymer chains as they can form coordination bonds with the negatively charged polymer chains to increase the cross-linking density of hydrogels. ${ }^{\mathbf{2 4}, 25}$ When an external loading is applied, these coordination bonds act as reversible sacrificial bonds and rupture to dissipate the applied energy. An improvement in tensile strength, elastic modulus, and fracture elongation was observed by adding $0.1 \mathrm{~mol} \% \mathrm{Al}^{3+}$ (Fig. 2). However, the mechanical properties of the hydrogels tend to remain stable with the addition of more than $0.5 \mathrm{~mol} \% \mathrm{Al}^{3+}$; in particular, within the $500 \%$ elongation, the stress-strain curves of Gel-0.5, Gel-1.0, and Gel-1.5 were almost coinciding. This phenomenon indicates that an excess of $\mathrm{Al}^{3+}$ concentration has little effect on the mechanical properties of the hydrogel since most of the carboxyl groups in the PAA chain are maintain in the acid form $(-\mathrm{COOH})$.

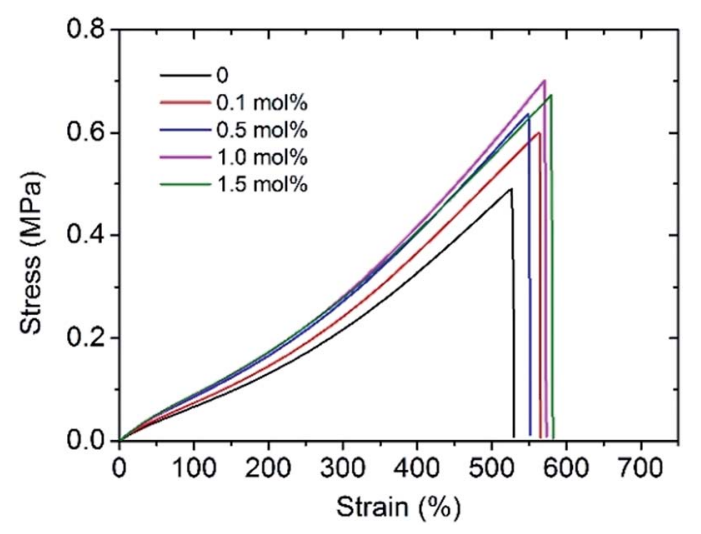

Fig. 2 Stress-strain curves of Gels with various $\mathrm{Al}^{3+}$ contents.
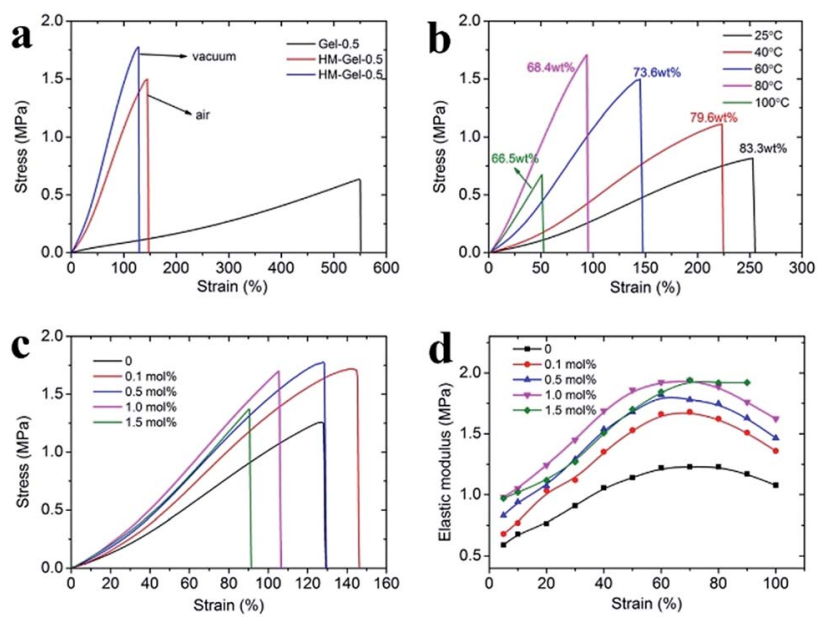

Fig. 3 (a) Stress-strain curves of Gel-0.5, HM-Gel-0.5, (dried in air at $60{ }^{\circ} \mathrm{C}$, red line; and evaporated in vacuum at $60{ }^{\circ} \mathrm{C}$, blue line); (b) stress-strain curves of $\mathrm{HM}$-Gel- 0.5 obtained at various evaporation temperatures, the water content of the swollen hydrogel at equilibrium is as indicated; (c) stress-strain curves of HM-Gels (dried in vacuum) with various $\mathrm{Al}^{3+}$ contents; (d) elastic modulus of the HMGels calculated from the derivative of the stress-strain curves in (c).

The hydrogels prepared by visible-light-triggered polymerization possessed remarkable mechanical properties (e.g., Gel0.5 , tensile strength of $0.66 \mathrm{MPa}$, strain of $550 \%$, and elastic modulus of $0.106 \mathrm{MPa}$ ). However, Gel-0.5, as well as many reported hydrogels, ${ }^{26,27}$ showed a lower elastic modulus as compared to some synthetic hydrogels (e.g., polyelectrolyte hydrogels $)^{28}$ or natural tissues (e.g., cartilage). ${ }^{29,30}$ In this study, we applied the evaporation-swelling (E-S) method on the asprepared Gel to achieve an approximately 10-fold increase in the elastic modulus of the hydrogel (Scheme 1). The mechanical properties of the as-prepared Gel underwent fundamental changes as a result of the restructuring of the hydrogel internal network. Fig. 3a shows that the E-S method-treated Gel (HM-Gel-0.5, air) reaches a high tensile strength and elastic modulus of 1.50 MPa and 0.96 MPa, respectively, which are 2.27 and 9 times higher than those of Gel-0.5. When the evaporation operation was conducted in vacuum, the HM-Gel-0.5 (vacuum) exhibited tensile strength $(1.78 \mathrm{MPa})$ and elastic modulus (1.29 MPa) that were 2.70 and 12.2 times higher than those of Gel-0.5, respectively (Fig. 3a). Apparently, the HM-Gels dried in vacuum displayed a higher tensile strength and elastic modulus than the HM-Gels dried in air, whereas a lower fracture elongation was found in the HM-Gels dried in vacuum. (Table $\mathrm{S} 1 \dagger$ ).

As shown in Fig. 3b, the evaporation temperature is a decisive factor for the mechanical performance of HM-Gels. When the evaporation proceeds at room temperature, the tensile strength and elastic modulus of HM-Gel-0.5 were only 1.24-fold and 1.83-fold, respectively, as compared to those of Gel-0.5. The tensile strength and elastic modulus of HM-Gel-0.5 dramatically increased with the increasing evaporation temperature, whereas the fracture elongation gradually decreased. This indicates that the evaporation temperature during the evaporation-swelling process is critical for the reconstruction of 
an internal network of the hydrogel. However, when Gel-0.5 was evaporated at $100{ }^{\circ} \mathrm{C}$ for $24 \mathrm{~h}$, the degeneration of the $\mathrm{CMC}$ network resulted in a substantial impairment of the mechanical properties. In addition, the evaporation temperature in the E-S method has a substantial effect on the water content of the hydrogel after the swelling equilibrium is reached. The water content of the hydrogel decreased from $83.3 \mathrm{wt} \%$ to $66.5 \mathrm{wt} \%$ as the evaporation temperature increased (Fig. 3b, inset labels).

Although the concentration of $\mathrm{Al}^{3+}$ has a limited effect on the mechanical properties of the as-prepared hydrogels, it is noted that the mechanical properties of HM-Gels can be greatly changed via the introduction of $\mathrm{Al}^{3+}$. The tensile strength increased to about 1.70 MPa upon increasing the $\mathrm{Al}^{3+}$ content from $0.1 \mathrm{~mol} \%$ to $1.0 \mathrm{~mol} \%$, whereas the fracture elongation gradually decreased. However, a higher $\mathrm{Al}^{3+}$ content (1.5 mol\%) could reduce the comprehensive mechanical properties of the HM-Gel (Fig. 3c). The elastic modulus was calculated from the derivative of the stress-strain curves of the HM-Gels. At a strain of $30 \%$, the elastic modulus of HM-Gels was in the range of 1.11-1.45 $\mathrm{MPa}$, depending on the $\mathrm{Al}^{3+}$ content, which was higher than $0.91 \mathrm{MPa}$ of HM-Gel without the reinforced crosslinking site $\left(\mathrm{Al}^{3+}\right)$. The elastic modulus of $\mathrm{HM}-\mathrm{Gel}-1.0$ was as high as $1.92 \mathrm{MPa}$ at a strain of $60 \%$ (Fig. 3d). The results indicate that an increase in $\mathrm{Al}^{3+}$ content can improve the tensile strength and elastic modulus of the hydrogels, whereas the fracture elongation first increased and then decreased. However, the addition of excess $\mathrm{Al}^{3+}$ reduces the mechanical properties of the hydrogel.

Both Gel and HM-Gel, like most tough hydrogels, are capable of withstanding large compressive stress. ${ }^{31}$ Fig. 4 shows that the compressive stress of Gel-0.5 and HM-Gel-0.5 can reach 17.1 MPa and 28.8 MPa, respectively, at a compressive strain of 90\%. HM-Gel-0.5 exhibited a higher compressive stress than other reported hydrogels under the same strain condition. ${ }^{\mathbf{2 6 , 3 2}}$ In the present study, the compressive modulus of HM-Gel-0.5 was always several times that of Gel-0.5, which should also be attributed to the recombination of the internal network structure of the hydrogel during the evaporation-swelling process.

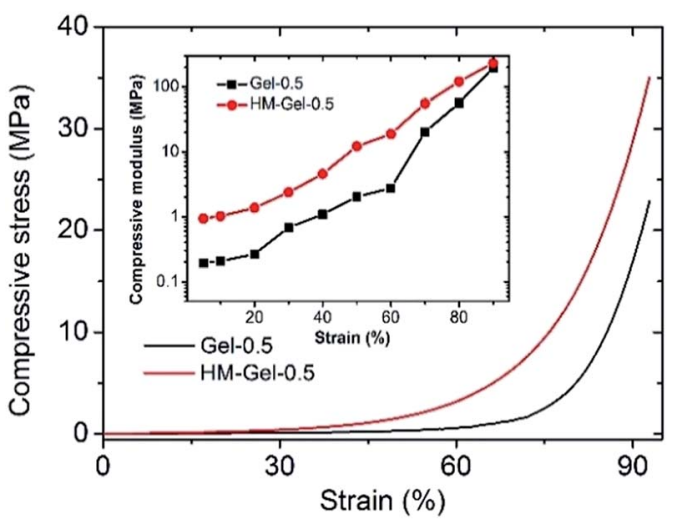

Fig. 4 Compressive stress-strain curves of Gel-0.5 and HM-Gel-0.5 (the calculated compressive modulus is shown in the inset in the figure).

\section{Internal structure evolution}

The conventional method for studying a hydrogel structure is to freeze-dry the hydrogel and then observe the pore structure of the hydrogel by an SEM. ${ }^{33}$ Although the basic skeleton structure of the hydrogel after freeze-drying is retained, its fine structure changes (e.g. the hydrogel changes from transparent to white). To investigate the actual changes occurring in the internal network of the hydrogel during the evaporation-swelling process, thin films $(200 \mu \mathrm{m})$ of these two kinds of hydrogel (Gel and HM-Gel) were obtained by shock-frozen section and observed using a polarized optical microscope in the wet state. In this hydrogel, some of the CMC are present in the aggregated state (crystalline cellulose) rather than completely in the form of molecules. Therefore, although the hydrogel is transparent, the internal network is heterogeneous. Due to the polarization property of crystalline cellulose ${ }^{34}$ we observed that a large amount of CMC was present in the form of crystals in the hydrogel (Fig. 5). In the as-prepared Gel (Fig. 5a), in addition to undissolved CMC chains exhibiting polarization property (black regions), the isolated CMC molecules involved in the polymerization reaction were not polarized (light-colored regions); this indicated that this CMC fraction was present in the noncrystalline form in the hydrogel. After the as-prepared Gel was treated through the E-S method (Fig. 5b), the edges of the crystalline CMC became irregular and many shadows (crystalline) filled in the light-colored regions as compared to the image shown in Fig. 5a. This indicated that the CMC crystal morphology and crystallinity had undergone tremendous changes. The images show that the E-S method greatly changes the hydrogel internal network of the hydrogel and increases the network's cross-linking density. The FT-IR spectra (Fig. S3†)

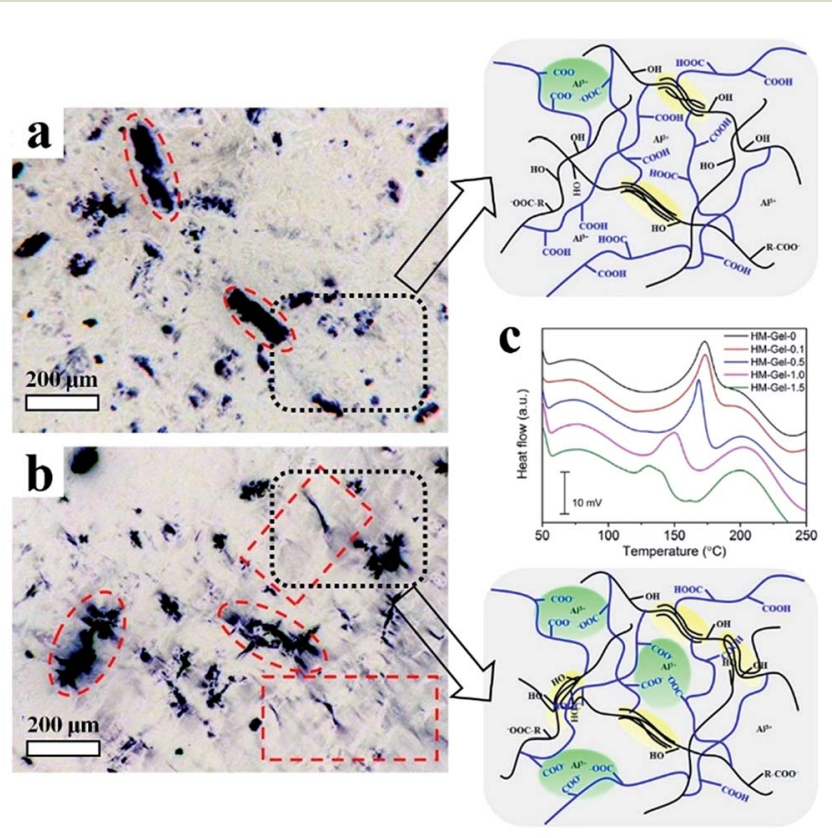

Fig. 5 ( $a$ and b) Images of Gel-0.5 and HM-Gel-0.5 films in the wet state under polarized light. The images are in negative mode for easy viewing; (c) effect of $\mathrm{Al}^{3+}$ content on the DTA curves of HM-Gels. 
showed that the absorption peaks of -COO-H in HM-Gel-0.5 were much stronger than those of PAA at $3000-3600 \mathrm{~cm}^{-1}$, indicating both PAA chains and CMC chains were involved in this reinforcement crystallization. In addition, the attenuation of the absorption peak of $1240 \mathrm{~cm}^{-1}(-\mathrm{CO}-\mathrm{OH})$ suggested that part of the carboxyl group and aluminum ion form more stable coordination compounds during this treatment process.

On the other hand, although the coordination bond could enhance the mechanical properties of the HM-Gel, the crystallinity of the HM-Gel was reduced. For example, an excessively high content of aluminum ions (as in HM-Gel-1.5) resulted in a very low crystallinity (the secondary crystallization region at only $75-130{ }^{\circ} \mathrm{C}$ ), which led to a decrease in mechanical properties (Fig. 5c). The calculated effective network chain density $(N)$ definitely confirmed that with the increase of $\mathrm{Al}^{3+}$ from 0 to $1 \mathrm{~mol} \%$, the $N$ increased from $82 \mathrm{~mol} \mathrm{~m}^{-3}$ to $130 \mathrm{~mol} \mathrm{~m}^{-3}$, whereas the excessively high amount of $\mathrm{Al}^{3+}$ led to the decrease of the $N$ (Fig. S4 $\dagger$ ). ${ }^{35}$ Therefore, by adjusting the content of aluminum ions to control the crystallinity of the HM-Gel, the required mechanical properties can be achieved.

\section{Swelling property}

Hydrogels, as soft and wet materials, consist of a cross-linked network and plenty of water (50-90\%) and have been commonly used in aqueous environments. ${ }^{36,37}$ The swelling behavior of hydrogels is very important for their practical applications. ${ }^{20}$ The swelling ratio $\left(Q_{\mathrm{m}}\right)$ of Gels decreased from 6.37 to 2.57 with the increase of $\mathrm{Al}^{3+}$ content. In sharp contrast, the $Q_{\mathrm{m}}$ of HM-Gels slightly changed from 1.22 to 0.97 in the same composition (Fig. 6). This large difference in the swelling ratios indicates that the crystallization induced by the E-S method severely hinders the swelling of the hydrophilic polymer network in distilled water.

\section{Viscoelasticity of the hydrogels}

In Fig. 7, the storage modulus $E^{\prime}$ and $\tan \delta$ are shown for the asprepared Gels and HM-Gels as functions of frequency at a strain of $1.0 \%$. The $E^{\prime}$ of all hydrogels increased as the frequency increased. Gel-0.5 had a higher $E^{\prime}$ than Gel-0 in the

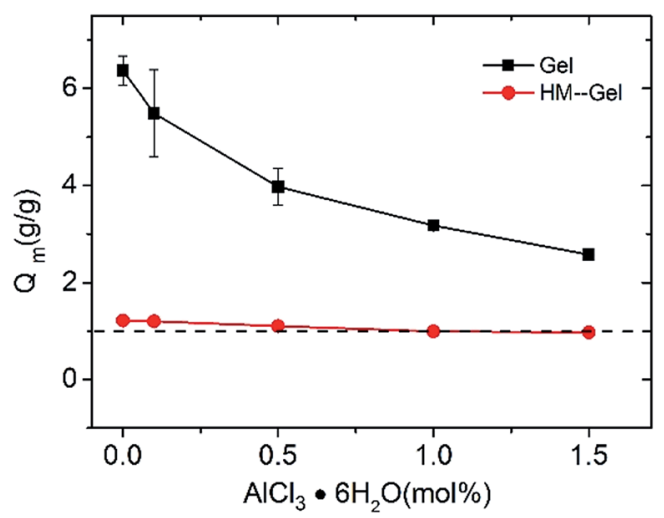

Fig. 6 Equilibrium swelling ratio $\left(Q_{m}\right)$ of the Gels and $H M$-Gels with various $\mathrm{Al}^{3+}$ contents. experimental frequency range; this suggested that the newly formed cross-linking site could increase the cross-linking network density by introducing $\mathrm{Al}^{3+} \cdot{ }^{38}$ The enhanced effect of $\mathrm{Al}^{3+}$ also occurred in HM-Gel, and the $\Delta E^{\prime}\left(E_{\text {Gel-0.5 }}^{\prime}-E_{\text {Gel-0 }}^{\prime}\right.$, and $E_{\text {HM-Gel-0.5 }}^{\prime}-E_{\text {HM-Gel-0) }}^{\prime}$ increased from $100 \mathrm{kPa}$ of Gel to more than $200 \mathrm{kPa}$ of HM-Gel at the frequency of $0.1-100 \mathrm{~Hz}$. These results confirm that the ions generated more coordination compounds, which increased the density of the cross-linked network during the evaporation-swelling process. The $E^{\prime}$ of the hydrogels with the same components (i.e., Gel-0 and HM-Gel-0 and Gel-0.5 and HM-Gel-0.5) were also different before and after the evaporation-swelling treatment. The increased $E^{\prime}$ indicates that the hydrogen bond recombination inside the hydrogel not only changes the structure of the crosslinked network, but also increases the cross-linking density of the network. The damping factor $(\tan \delta)$ is determined by the storage modulus and loss modulus ratio. ${ }^{39}$ Unlike the storage modulus, the $\tan \delta$ value of hydrogels with the same composition was reduced before and after treatment via the E-S method; this may be because the increased cross-linking density limited the movement of partial polymer chains. The $\tan \delta$ value of all hydrogels showed a clear critical frequency at approximately $25 \mathrm{~Hz}$ (Fig. 7b and S5†) and increased rapidly as the frequency
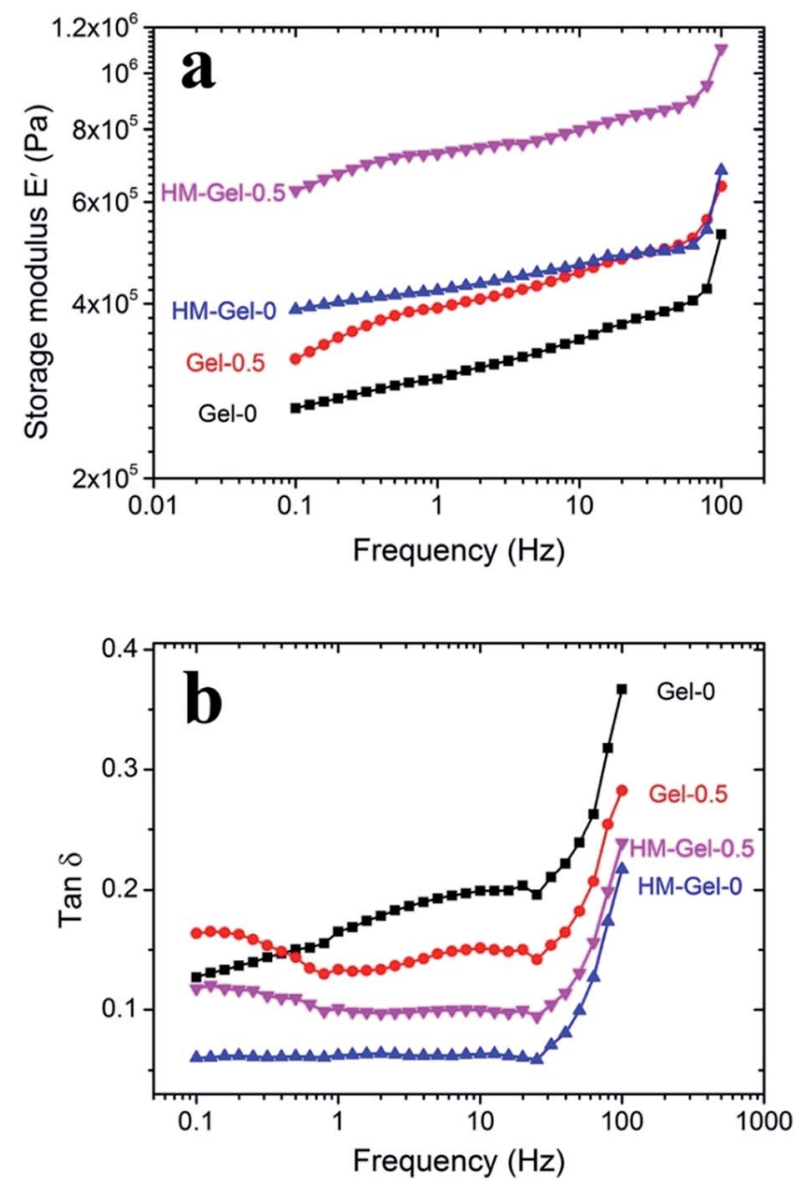

Fig. 7 Dynamic mechanical analysis of the hydrogels: (a) storage modulus $\left(E^{\prime}\right)$ and $(b)$ damping factor $(\tan \delta$ ) of Gel-0, HM-Gel-0, Gel0.5, and HM-Gel-0.5. 
increased; this indicated that the energy dissipation caused by intermolecular friction was proportional to the frequency. For the hydrogel without $\mathrm{Al}^{3+}$, the $\tan \delta$ value of HM-Gel-0 was significantly lower than that of Gel-0 after evaporation-swelling treatment. However, with the introduction of a certain amount of $\mathrm{Al}^{3+}$ into the hydrogel, the decrease in $\tan \delta$ value of the hydrogel containing $\mathrm{Al}^{3+}$ before and after the evaporationswelling treatment was significantly smaller than that of the aluminum-free hydrogel. Interestingly, the $\tan \delta$ of Gel-0.5 and HM-Gel-0.5 had another critical frequency (approximately $1 \mathrm{~Hz}$ ); this phenomenon could also be observed in the hydrogel with a higher $\mathrm{Al}^{3+}$ content (over $0.5 \mathrm{~mol} \% \mathrm{Al}^{3+}$, Fig. S5†). This change in the frequency response may be due to the rupture of ionic bonds within the hydrogel containing $\mathrm{Al}^{3+}$. Therefore, the damping characteristic of hydrogels contributes to their potential application in the field of vibration absorption.

\section{Cyclic mechanical property}

The HM-Gel exhibited excellent tensile and compressive properties during successive cyclic process. Fig. 8a shows that the HM-Gel-0.5 has basic stable stress-time curves. However, it should be noted that the maximum stress for HM-Gel-0.5 slightly decreased from $0.56 \mathrm{MPa}$ at an initial time and then trended to remain unchanged at $0.50 \mathrm{MPa}$. This may be due to the fact that during the stretching process, the hydrogel network obtained by free radical polymerization generally undergoes rupture and sliding of the polymer chains as well as rupture of ionic and hydrogen bonds, according to a previous study. ${ }^{16}$ In addition, the slight sliding between the hydrogel and the fixture also contributes to decreasing the maximum stress. ${ }^{40}$ After the stretched hydrogel was stored at room temperature for 24 hours in a sealed and humid environment, the sample exhibited very similar stress-time curves to the previous curves. The similarity between the curves indicated that the ionic and hydrogen bonds in the Gel were substantially healed. ${ }^{41}$ The only non-coincident part was the curve of the first loading process, confirming that the irreversible rupture and permanent sliding of the partial polymer chains only occurred during the first stretching process. ${ }^{16} \mathrm{Fig}$. $8 \mathrm{~b}$ shows the cyclic compression curves obtained after 10 successive tests at various strains. At a lower strain (less than $25 \%$ ), the compression loading-unloading curves were always consistent; this indicated that the internal network structure of HM-Gel-0.5 was retained. However, as the compressive strain increases, the network structure of HM-Gel-0.5 changes. At a strain of $50 \%$ and $70 \%$, the curves of the second cycle were inconsistent with those of the first cycle; this suggested that a large deformation may cause irreversible damage to the network structure. At a strain of $25 \%$, $50 \%$, and $70 \%$ (from the second cycle), the hydrogels displayed a stable hysteresis energy dissipation, which resulted from the rupture of ionic and hydrogen bonds and the friction between polymer chains. Thus, the HM-Gel is a suitable candidate for vibration absorption materials.

\section{Vibration absorption of the hydrogel}

To verify the actual vibration absorption effect of the hydrogel, we tested the vibrational absorption of the hydrogel (HM-Gel-
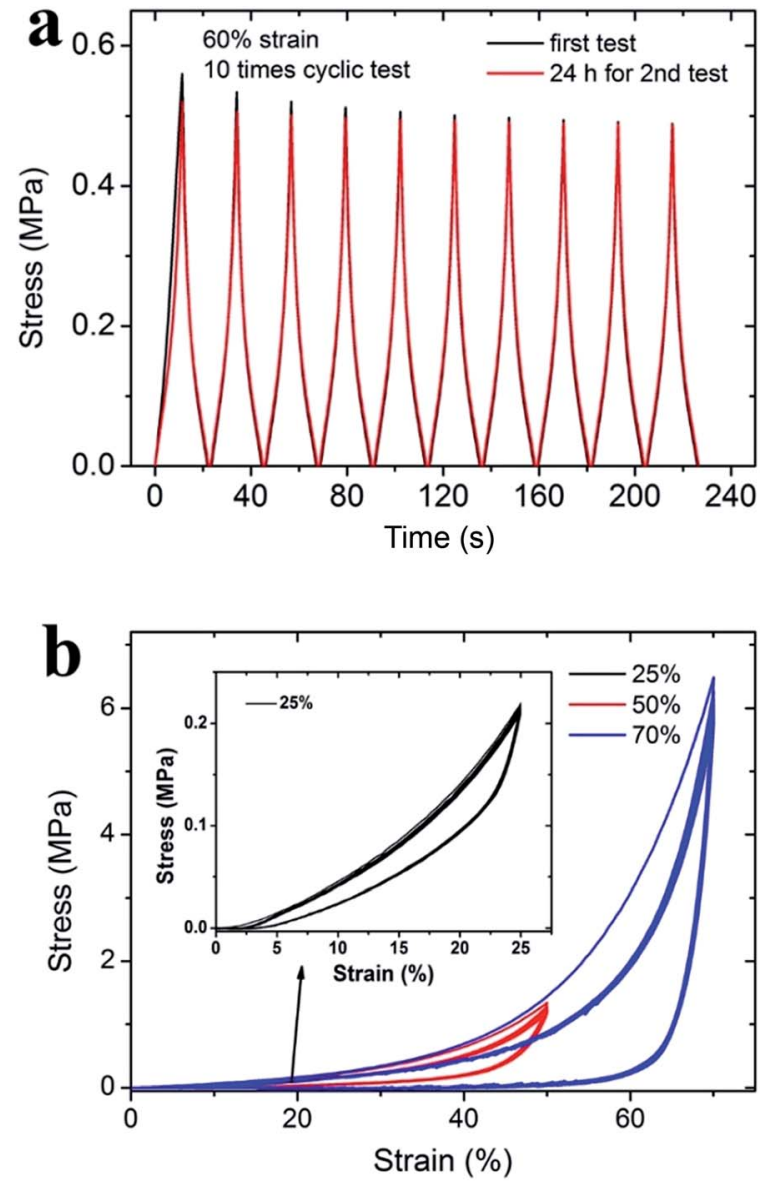

Fig. 8 (a) Cyclic stress-time curves of HM-Gel-0.5 obtained after 10 tests at a strain of $60 \%$, and the same operation was performed after $24 \mathrm{~h}$; (b) cyclic compression curves of HM-Gel-0.5 obtained after 10 tests at various strains.

0.5) using the commercial test tube mixer (Present Mixer 2013, remove the spring bracket, Fig. 9). The test tube mixer was vigorously vibrated on a metal plate and submitted to a large displacement without any cushioning (Movie S1†). When the test tube mixer was placed on the hydrogel, the machine was able to operate stably on the hydrogel after it experienced a partial initial displacement (caused by resonance). Thus, the hydrogel was able to efficiently absorb the vibration and stabilize the test tube mixer (Movie S2 $\dagger$ ). The detected vibration forces of Gel and HM-Gel were $1.4 \mathrm{~N}$ and $0.6 \mathrm{~N}$, respectively, which were lower than the vibration force of the machine without a vibration damper $(8.5 \mathrm{~N})$. In a vibration absorption material, stiffness is an important performance index, and a high stiffness can improve the material's vibration absorption performance. ${ }^{8,42}$

The stiffness is defined as

$$
k=\frac{F}{\delta}
$$

or

$$
k=\frac{A E}{l}
$$




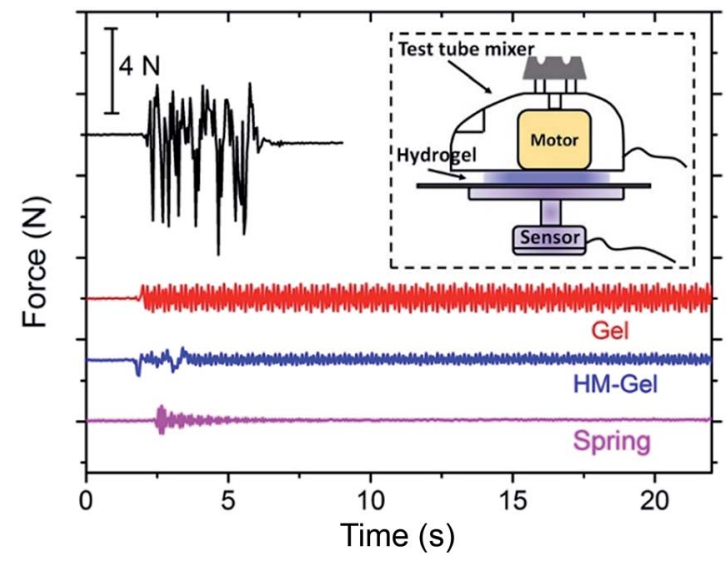

Fig. 9 Comparison of the vibration absorption effect obtained with different vibration dampers in free mode.

where $F$ is the force on the body, $\delta$ is the displacement along the force, $A$ is the cross-sectional area, $E$ is the elastic modulus, and $l$ is the length of the element.

For the specific case of an unconstrained uniaxial tension or compression, the elastic modulus can be thought of as a measure of the stiffness of a structure. Thus, the highmodulus hydrogel (HM-Gel-0.5, with an elastic modulus of approximately $1 \mathrm{MPa}$ ), obtained by the E-S method, can withstand a greater load under the unit strain than the low-modulus hydrogel (Gel-0.5, with an elastic modulus of approximately $0.1 \mathrm{MPa}$ ) and showed a better vibration absorption performance (Fig. 9). Of course, the metal spring bracket displayed the best vibration absorption.

\section{Conclusions}

In summary, we first synthesized a hydrogel (Gel) with good mechanical properties via a visible-light-trigger polymerization. To increase the modulus of the hydrogel, such that this can be applied as vibration absorption material, we leveraged the hydrogen bonds in the hydrogel to induce a CMC crystallization to alter the network structure within the hydrogel via the E-S method. The obtained optimal HM-Gel possessed an excellent mechanical properties with tensile strength and elastic modulus of 1.55 MPa and 1.02 MPa, respectively. Furthermore, different evaporation temperatures and the added various aluminum ion concentration have an extremely important effect on the tensile strength, modulus, and swelling ratio of the hydrogel. The coordination bonds formed by aluminum ions and anionic polymers increased the damping in the hydrogel during vibration, thus improving the vibration absorption performance of the hydrogel. In addition, HM-Gel-0.5 exhibited stable mechanical properties in both the cyclic tensile and compression tests. The preliminary application experiment shows that the high-modulus hydrogel has a better vibration absorption effect than the low-modulus hydrogel. We hope that this design concept can enhance the modulus of more hydrogels containing hydrogen bonds, such that these hydrogels can be used not only as machinery vibration absorbers but also as biological vibration absorbers (for example: articular cartilage).

\section{Conflicts of interest}

There are no conflicts to declare.

\section{Acknowledgements}

This research was supported by the International Collaboration Project funded by the Shenzhen Government (GJHZ20150316160614843). L. Zhu would like to acknowledge the China Scholarship Council for the Ph.D. program.

\section{Notes and references}

1 E. S. Dragan, Chem. Eng. J., 2014, 243, 572-590.

2 X. Zhao, Soft Matter, 2014, 10, 672-687.

3 T. Nonoyama, S. Wada, R. Kiyama, N. Kitamura, M. T. Mredha, X. Zhang, T. Kurokawa, T. Nakajima, Y. Takagi, K. Yasuda and J. P. Gong, Adv. Mater., 2016, 28, 6740-6745.

4 A. M. Hopkins, L. De Laporte, F. Tortelli, E. Spedden, C. Staii, T. J. Atherton, J. A. Hubbell and D. L. Kaplan, Adv. Funct. Mater., 2013, 23, 5140-5149.

5 W. Huang, A. Tarakanova, N. Dinjaski, Q. Wang, X. Xia, Y. Chen, J. Y. Wong, M. J. Buehler and D. L. Kaplan, Adv. Funct. Mater., 2016, 26, 4113-4123.

6 A. M. S. Costa and J. F. Mano, Eur. Polym. J., 2015, 72, 344364.

7 S. Lv, D. M. Dudek, Y. Cao, M. M. Balamurali, J. Gosline and H. Li, Nature, 2010, 465, 69-73.

8 D. D. L. Chung, J. Mater. Sci., 2001, 36, 5733-5737.

9 G. Gao, G. Du, Y. Sun and J. Fu, ACS Appl. Mater. Interfaces, 2015, 7, 5029-5037.

10 S. Liu and L. Li, ACS Appl. Mater. Interfaces, 2016, 8, 2974929758.

11 C. W. Peak, J. J. Wilker and G. Schmidt, Colloid Polym. Sci., 2013, 291, 2031-2047.

12 T. L. Sun, T. Kurokawa, S. Kuroda, A. B. Ihsan, T. Akasaki, K. Sato, M. A. Haque, T. Nakajima and J. P. Gong, Nat. Mater., 2013, 12, 932-937.

13 P. Lin, S. Ma, X. Wang and F. Zhou, Adv. Mater., 2015, 27, 2054-2059.

14 Y. Yang, X. Wang, F. Yang, H. Shen and D. Wu, Adv. Mater., 2016, 28, 7178-7184.

15 L. Zhu, J. Qiu, E. Sakai, L. Zang, Y. Yu, K. Ito, P. Liu and F. Kang, Macromol. Mater. Eng., 2017, 302, 1600509.

16 L. Zhu, J. Qiu, E. Sakai and K. Ito, ACS Appl. Mater. Interfaces, 2017, 9, 13593-13601.

17 N. J. Treat, B. P. Fors, J. W. Kramer, M. Christianson, C.-Y. Chiu, J. Read de Alaniz and C. J. Hawker, ACS Macro Lett., 2014, 3, 580-584.

18 X. Dai, Y. Zhang, L. Gao, T. Bai, W. Wang, Y. Cui and W. Liu, Adv. Mater., 2015, 27, 3566-3571. 
19 L. Fakhru, A. Razi, I. Y. Qudsieh, W. M. Z. W. Yunus, M. B. Ahmad and M. Z. A. Rahman, J. Appl. Polym. Sci., 2001, 82, 1375-1381.

20 H. Kamata, Y. Akagi, Y. Kayasuga-Kariya, U. I. Chung and T. Sakai, Science, 2014, 343, 873-875.

21 F. Luo, T. L. Sun, T. Nakajima, T. Kurokawa, Y. Zhao, K. Sato, A. B. Ihsan, X. Li, H. Guo and J. P. Gong, Adv. Mater., 2015, 27, 2722-2727.

22 M. Guo, L. M. Pitet, H. M. Wyss, M. Vos, P. Y. Dankers and E. W. Meijer, J. Am. Chem. Soc., 2014, 136, 6969-6977.

23 W. J. Zheng, N. An, J. H. Yang, J. Zhou and Y. M. Chen, ACS Appl. Mater. Interfaces, 2015, 7, 1758-1764.

24 C. H. Yang, M. X. Wang, H. Haider, J. H. Yang, J. Y. Sun, Y. M. Chen, J. Zhou and Z. Suo, ACS Appl. Mater. Interfaces, 2013, 5, 10418-10422.

25 J. Y. Sun, X. Zhao, W. R. Illeperuma, O. Chaudhuri, K. H. Oh, D. J. Mooney, J. J. Vlassak and Z. Suo, Nature, 2012, 489, 133136.

26 Q. Chen, L. Zhu, C. Zhao, Q. Wang and J. Zheng, Adv. Mater., 2013, 25, 4171-4176.

27 M. M. Fitzgerald, K. Bootsma, J. A. Berberich and J. L. Sparks, Biomacromolecules, 2015, 16, 1497-1505.

28 J. You, S. Xie, J. Cao, H. Ge, M. Xu, L. Zhang and J. Zhou, Macromolecules, 2016, 49, 1049-1059.

29 S. Pal, Design of Artificial Human Joints \& Organs, Mechanical Properties of Biological Materials, 2014, ch. 2, pp. 23-40.
30 Z. Wang, F. Tao and Q. Pan, J. Mater. Chem. A, 2016, 4, 17732-17739.

31 G. Du, L. Nie, G. Gao, Y. Sun, R. Hou, H. Zhang, T. Chen and J. Fu, ACS Appl. Mater. Interfaces, 2015, 7, 3003-3008.

32 J. Duan, X. Liang, Y. Cao, S. Wang and L. Zhang, Macromolecules, 2015, 48, 2706-2714.

33 J. Fang, A. Mehlich, N. Koga, J. Huang, R. Koga, X. Gao, C. Hu, C. Jin, M. Rief, J. Kast, D. Baker and H. Li, Nat. Commun., 2013, 4, 2974.

34 R. J. Moon, A. Martini, J. Nairn, J. Simonsen and J. Youngblood, Chem. Soc. Rev., 2011, 40, 3941-3994.

35 J. Yang, C. R. Han, J. F. Duan, F. Xu and R. C. Sun, ACS Appl. Mater. Interfaces, 2013, 5, 3199-3207.

36 S. Pina, J. M. Oliveira and R. L. Reis, Adv. Mater., 2015, 27, 1143-1169.

37 J. Kim, A. Conway and A. Chauhan, Biomaterials, 2008, 29, 2259-2269.

38 Z. Cai, D. H. Kwak, D. Punihaole, Z. Hong, S. S. Velankar, X. Liu and S. A. Asher, Angew. Chem., 2015, 54, 13036-13040.

39 D. Shanmugam and M. Thiruchitrambalam, Mater. Des., 2013, 50, 533-542.

40 M. Lillie, G. Chalmers and J. Gosline, Connect. Tissue Res., 1994, 31, 23-35.

41 M. Zhong, X. Y. Liu, F. K. Shi, L. Q. Zhang, X. P. Wang, A. G. Cheetham, H. Cui and X. M. Xie, Soft Matter, 2015, 11, 4235-4241.

42 R. Lakes, J. Compos. Mater., 2002, 36, 287-297. 